\title{
A Projection And Clipping Method To Calculate Direct, Diffuse, And Reflected Irradiation
}

\author{
Jacob Estevam Schmiedt, Björn Schiricke \\ German Aerospace Center (DLR), Cologne, Germany
}

\begin{abstract}
Various approaches are used to determine direct, sky diffuse, and reflected contributions to the solar irradiance on building surfaces. Among these are ray tracing, pixel counting, and projection and clipping. Projection and clipping is closest to an exact solution but it is often considered as too slow.

This paper presents an improved projection and clipping method to determine the direct and the sky diffuse contribution to the irradiance. In a second step a projection and clipping approach is applied to calculate the view factors between building surfaces, so that the reflected contribution can be obtained.

The presented approach delivers results which are comparable to those obtained by EnergyPlus in acceptable computation time. This shows that modern building simulation tools can benefit from an approach that applies fewer approximations than common raytracing or pixel counting techniques.
\end{abstract}

\section{Introduction}

Accurate data of the expected solar irradiation on building envelops is crucial information to reduce the use of fossil energy by buildings. Integrated irradiation for longer time spans such as months or years is required to assess the potential of solar energy generation. With the ongoing development of facadeintegrated photovoltaics (PV) also the vertical parts of buildings are becoming relevant when the potential for PV and solar heat generation is studied (Redweik et al. (2013), Catita et al. (2014)). Higher time resolutions, such as hourly values, are needed to determine external loads for dynamic energy simulations of buildings or districts.

The calculation of direct solar irradiation on a tilted surface without shading is a simple exercise. However, it is a much more complex task to determine irradiances for surfaces which may be shaded by various objects and to include the diffuse contribution. The diffuse solar irradiation consists of two part: a sky diffuse part due to scattering of light on particles in the atmosphere and a contribution from reflections on the ground and surrounding objects. In dense ur- ban areas shading and reflections have a strong influence on the energy performance of buildings (Han et al. (2017)) and accurate methods to determine all contributions to the solar irradiance are required.

In the literature and in existing software solutions various approaches exist to determine shading, sky diffuse and reflected irradiation on building surfaces. The simulation software RADIANCE is widely considered an advanced tool for irradiation and illumination modeling (Freitas et al. (2015); Liao and Heo (2017)). It uses a backward-raytracing approach and accounts for diffuse as well as specular reflections. Backward-raytracing techniques choose a finite number of receiving points on a receiving surface. From each point rays are sent out into a hemisphere and their intersection points with other surfaces or volumes is calculated. Rays that do not intersect with any objects reach the sky dome, so that the surface is irradiated directly by the direct or sky diffuse radiation under the corresponding solid angle.

Projection an clipping ( $\mathrm{P} \& \mathrm{C})$ approaches are especially successful when the shading of direct solar irradiation is determined. The building envelope is described by polygons and a combination of rotation, projection, and boolean operations (intersection, difference, union) is used to calculate the sunlit and shaded parts of the building hull. While raytracing works with a finite number of starting points for the rays, $\mathrm{P} \& \mathrm{C}$ does not apply any approximation or interpolation to determine the shading of direct irradiation. The development of $\mathrm{P} \& \mathrm{C}$ methods goes back several decades (Atherton et al. (1978); Maestre et al. (2013); Melo et al. (2013)). In combination with a discretization of the sky dome it is also used to determine the sky diffuse irradiation, e.g. by EnergyPlus (US DOE (2007)). While P\&C requires fewer approximations than raytracing its calculations are usually more time-consuming.

In recent years also pixel counting techniques have gained some attention. These techniques calculate the sunlit and shaded parts of building surfaces by counting the number of pixels that are visible from a certain vantage point. Thus, they are not ex- 
act but often perform better than $\mathrm{P} \& \mathrm{C}$ approaches (Niewienda and Heidt (1996); de Almeida Rocha et al. (2017)).

A widely used and well tested software tool for building energy simulation is EnergyPlus (Crawley et al. (2008); Kim and Kim (2015)) which will serve as the reference software in this paper. It uses a combination of $\mathrm{P} \& \mathrm{C}$ and raytracing to determine the full irradiance on building surfaces. A P\&C technique is used to calculate the direct and sky diffuse contribution to the irradiance while raytracing is used to determine the reflected contribution.

With the increasing availability and decreasing cost of remote sensing technology city-wide 3D building models are becoming widely available. They are usually obtained by LIDAR or photogrammetry (Verma et al. (2006); Frommholz et al. (2015)). Common formats, e.g. CityGML (OGC (2012)), use polygons with semantical annotation to represent the buildings and their environment. Therefore, a polygonbased approach like the $\mathrm{P} \& \mathrm{C}$ technique appears to be a natural way to determine solar irradiation on building surfaces.

In this paper an approach to determine irradiances on building envelope surfaces is presented that relies only on $\mathrm{P} \& \mathrm{C}$ to obtain direct, sky diffuse, and reflected contributions to the irradiance on building surfaces. In the following it will be termed "refined P\&C" approach. In this paper it is shown that there is still room for improvement of the performance of P\&Cbased approaches. Furthermore, it is shown that the advantage that $\mathrm{P} \& \mathrm{C}$ techniques require only few approximations can be exploited also for the calculation of reflections. For the direct and sky diffuse contribution it builds on the procedure proposed by Maestre et al. (2013). Maestre et al. (2013) explain how several exclusion rules are used to select possibly shading surface for every receiving surface. Here, another condition is introduced that further reduces the number of of possibly shading surfaces and, thus, reduces the computational effort. Information about surfaces that may shade a receiving surface is also used to narrow down the number of possibly reflecting surfaces. The approach is applied to a reference scenario which consists of a hypothetical building ensemble with four buildings. The surfaces of these buildings obstruct solar radiation and reflect it. The results for this are compared to the outcome of calculations in EnergyPlus for the same scenario.

The presented work is part of ongoing research to make remote sensing techniques applicable for energetic analyses of buildings (Estevam Schmiedt et al. (2017)). Its goal is to develop a tool box of measurement and analysis methods to determine the thermal properties of building envelopes quickly and accurately. Thus, single buildings or whole districts may be examined in short time to obtain crucial informa- tion for the development of refurbishment strategies or about loads of the energy networks.

\section{Methods}

The Method presented in this paper consists of three parts: The discretization of a hemisphere with the allocation of radiances to the sky patches, a projection and clipping procedure to calculate the direct and sky diffuse irradiance on the building envelops, and a subsequent calculation of the reflected contribution to the irradiance on the building envelop.

\section{Sky model and discretization of the hemi- sphere}

The solar irradiance coming from the sky can generally be divided into two parts: A direct and a diffuse contribution. The direct part depends on the day of the year, the time of the day, the location, and the atmospheric conditions. The diffuse part is due to scattering of solar light on the particles in the atmosphere. Various models exist that connect the direct and the diffuse radiance (Perez et al. (1990, 1993); Igawa et al. (2004)). In this paper both contributions are superimposed and treated together when the irradiance for building surfaces is calculated.

In this paper the model developed by Perez et al. (1993) is used to calculate the angle dependence of the luminance distribution $l(\theta, \gamma)$ due to diffuse solar radiation. The photometric quantity luminance is then converted into irradiance using a normailzation factor. Its distribution is a function of the zenith angle of the sun $\theta$ and the angle $\gamma$ between the considered sky element and the position of the sun. The model by Perez et al. (1993) uses the diffuse horizontal irradiance, the direct normal irradiance, the extraterrestrial direct normal irradiance, and the position of the sun as input. The irradiance values have to be obtained from modeled or measured weather data as it is provided, e.g., with hourly resolution by the IWEC Weather Files ASHRAE (2001) for typical meteorological years of many locations in Europe. The Python library Pysolar which computes the location of the sun based on Bretagnon's VSOP 87 theory (Bretagnon and Francou (1988)) is used to obtain the zenith angle $\theta$ and azimuth angle $\varphi$ of the sun.

While the luminance is a useful quantity to model daylighting, the calculation of possible heat gains due to solar irradiation or the potential for solar energy requires radiance values. A common approach (Robinson and Stone (2004b)) to obtain the sky diffuse contribution to the radiance is to define a normalization factor

$$
\chi=I_{\mathrm{dh}} / \int_{0}^{2 \pi} \int_{0}^{\pi / 2} l(\theta, \gamma) \cdot \cos (\pi / 2-\theta) d \Omega,
$$

where $I_{\mathrm{dh}}$ is the diffuse horizontal irradiance. The angle dependent diffuse radiance is then

$$
R_{\mathrm{d}}(\theta, \gamma)=l(\theta, \gamma) \chi .
$$


For numerical purposes the upper hemisphere has to be discretized. Here a similar approach for its subdivision as in Robinson and Stone (2004a) is used. This means the upper hemisphere is discretized into 151 patches according to Tregenza (1987). The diffuse radiance $R_{\mathrm{d}}\left(\theta_{k}, \varphi_{k}\right)$ is calculated with the Perez model for the center of every patch. The radiance is assumed to be constant for the whole patch. Therefore, the corresponding unobstructed normal irradiance for a patch with the center at $\left(\theta_{k}, \varphi_{k}\right)$ is $I_{\mathrm{d}}^{\mathrm{n}}\left(\theta_{k}, \varphi_{k}\right)=R_{\mathrm{d}}\left(\theta_{k}, \varphi_{k}\right) \Delta \Omega_{k}$, where $\Delta \Omega_{k}$ is the solid angle covered by patch $k$.

To obtain the total radiance distribution the patch center with smallest $\gamma$ is determined for each point in time and the direct normal irradiance is added to its diffuse irradiance. The irradiance values for all patches can be calculated and stored for each time step or integrated over a given period of time, e.g. one year.

\section{Shading calculations}

The refined $\mathrm{P} \& \mathrm{C}$ approach to determine the direct and sky diffuse irradiance on the building surfaces is based on the setup of an irradiation matrix to speed up the calculation of irradiances with high time resolution. For a single patch center $\left(\theta_{k}, \varphi_{k}\right)$ the irradiance on a surface $n$ without shading is given by

$$
I_{n, k}^{0}=\mathbf{n}_{n} \cdot \mathbf{n}_{k}^{\text {sky }} I^{n}\left(\theta_{k}, \varphi_{k}\right),
$$

where

$$
\mathbf{n}_{k}^{\text {sky }}=-\left(\begin{array}{c}
\cos \varphi_{k} \sin \theta_{k} \\
\sin \varphi_{k} \sin \theta_{k} \\
\cos \theta_{k}
\end{array}\right) .
$$

$\mathbf{n}_{n}$ is the normal vector of the surface which points out of the building.

To construct the irradiation matrix the shaded and the lit area on every building surface must be determined for every $\left(\theta_{k}, \varphi_{k}\right)$. For this purpose an approach based on a procedure which has been proposed by Maestre et al. (2013) is used. This means that the polygons which describe the building surfaces are projected onto a plane perpendicular to $\mathbf{n}_{k}^{\text {sky }}$ and several boolean operations (intersection, difference, union) are performed to calculate the shadows that the polygons cast onto each other. These operations are also termed "clipping" operations. To reduce the number of clipping operations Maestre et al. (2013) describe certain exclusion conditions which narrow down the number of surfaces that may shade a receiving surface. E.g., looking into the direction of $\mathbf{n}_{k}^{\text {sky }}$, all surfaces that lie behind the receiving surface can be excluded from the clipping. The operations are then performed only between the receiving surface and this limited number of possibly shading surfaces. In the work presented here, an additional speed-up of the calculation is achieved when the condition is used that for shading surfaces

$$
\mathbf{n}_{n} \cdot \mathbf{n}_{k}^{\text {sky }}<0
$$
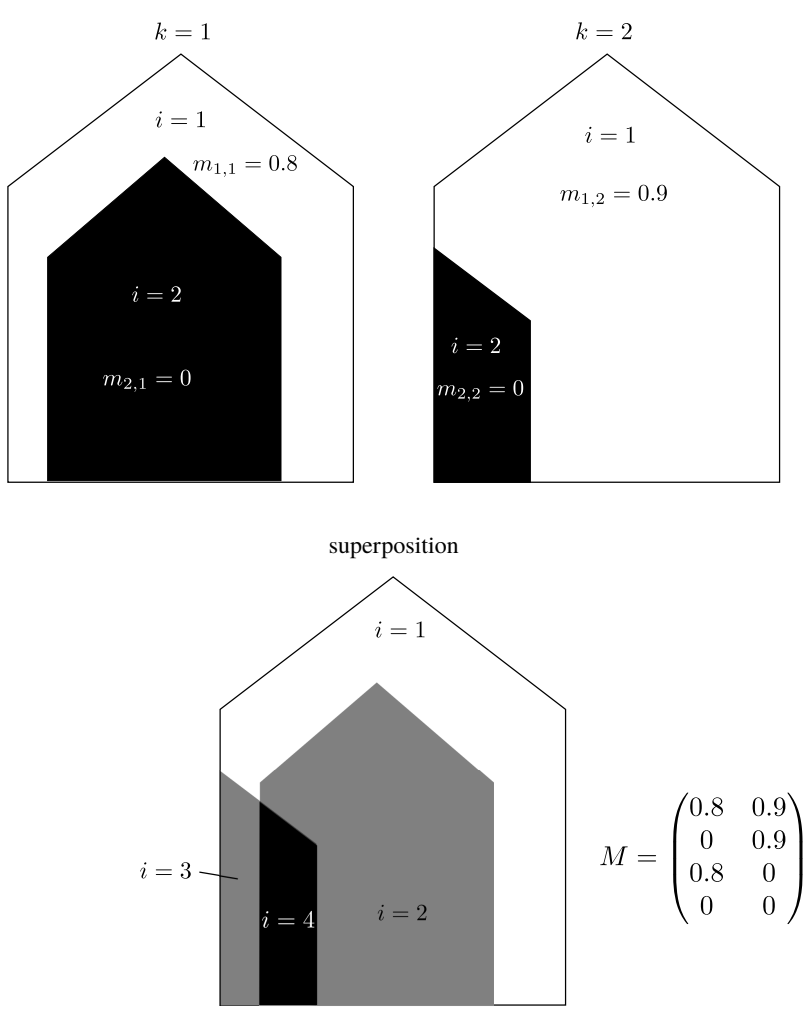

Figure 1: Illustration of the superposition of polygons obtained for two different $\left(\theta_{k}, \varphi_{k}\right) . M$ is the irradiation matrix after this superposition.

must be fulfilled if they are part of a volume object. For every volume object the union of shadows that the set of surfaces with $\mathbf{n}_{n} \cdot \mathbf{n}_{k}^{\text {sky }}<0$ cast onto a plane perpendicular to $\mathbf{n}_{k}^{\text {sky }}$ and the union of shadows by the set of surfaces with $\mathbf{n}_{n} \cdot \mathbf{n}_{k}^{\text {sky }}>0$ coincide. Therefore, the $\mathrm{P} \& \mathrm{C}$ operations have to be performed only for one of the two sets. An irradiation factor $m_{i, k}$ is attributed to each polygon that is produced by the clipping operations. It can be $m_{i, k}=0$ for a shaded polygon or $m_{i, k}=\mathbf{n}_{n(i)} \cdot \mathbf{n}_{k}^{\text {sky }}$, where $n(i)$ is the index of the surface that the polygon $i$ belongs to. If $\mathbf{n}_{n(i)} \cdot \mathbf{n}_{k}^{\text {sky }}<0$ the sun shines onto the back of a polygon, so that the front remains dark. Therefore, $m_{i, k}$ is set to zero.

After calculating shaded and irradiated polygons on the building surfaces for every patch the superposition of the polygons is calculated with another round of $\mathrm{P} \& \mathrm{C}$ operations. The following procedure is performed for each surface separately.

1. For every $\left(\theta_{k}, \varphi_{k}\right)$ the polygons are expressed in a coordinate system in which the $z$ direction coincides with the norm of the surface. Afterwards they are projected onto the plane with $z=0$ in the new coordinate system.

2 . The projected polygons for different $\left(\theta_{k}, \phi_{k}\right)$ are superimposed subsequently as it is shown for the first two $k$ in figure 1 . The indices of the surfaces 
that shade the polygon $i=2$ for $k=1$ is stored together with the polygons $i=2$ and $i=4$ in the superposition. Likewise, the indices of the surfaces that shade $i=2$ for $k=2$ are stored together with the polygons $i=3$ and $i=4$.

3 . The superposition of the first two $k$ is superimposed in the same way with the third. In this way a superposition of all polygons for all $\left(\theta_{k}, \varphi_{k}\right)$ is obtained. The irradiation matrix is successively created as indicated in figure 1 .

4. The value of the $z$ coordinate is added for all vertices and they are expressed in the original coordinate system.

This procedure gives a set of polygons for every surface with annotated information that contains the angle under which a shadow is cast onto it and the surfaces that cast shadows on it. It also gives the irradiation matrix $M$. The irradiances for every polygon are then calculated by

$$
I_{i}=\sum_{k} M_{i, k} I^{n}\left(\theta_{k}, \varphi_{k}\right)
$$

The irradiances of the original building surfaces are obtained by an area-weighted average. $I^{n}\left(\theta_{k}, \varphi_{k}\right)$ is the only quantity here that changes over time.

\section{Reflections}

The calculation of reflections benefits from the previous caclulations. Only diffuse reflections are considered and the surfaces are assumed to be the surfaces of ideal grey bodys. Radiation is reflected multiple times from surface to surface. With every reflection a part of the radiation is absorbed so that the dominant contribution is the term which describes a single reflection and is first order in the reflectance. The irradiance on polygon $i$ due to single reflections from the other surfaces is given by

$$
I_{i}^{\mathrm{refl}}=\sum_{j \neq i} F_{i j} \rho_{j} I_{j} A_{j},
$$

where $A_{j}$ is the area of polygon $j, F_{i, j}$ is the view factor from $i$ to $j$, and $\rho_{j}$ is the reflectance of polygon $j$. The view factor is defined as

$$
F_{i j}=\frac{1}{\pi A_{i}} \int_{A_{i}} \int_{A_{j}} \frac{\cos \alpha_{i} \cos \alpha_{j}}{r^{2}} \nu_{i, j} \mathrm{~d} A_{i} \mathrm{~d} A_{j},
$$

where the function $\nu_{i, j}$ is one when the differential area $i$ can "see" the differential area $j$, i.e. the view onto area $j$ is not obstructed by any other surface, or zero when it cannot. Figure 2 illustrates the definitions of $\alpha_{i}, \alpha_{j}$, and $r$.

For the numerical calculation of the view factors the full solid angle is again subdivided into patches. The same subdivision that is used for the sky dome is applied for the hemisphere which is visible from a polygon. An infinitesimal view factor between an in-

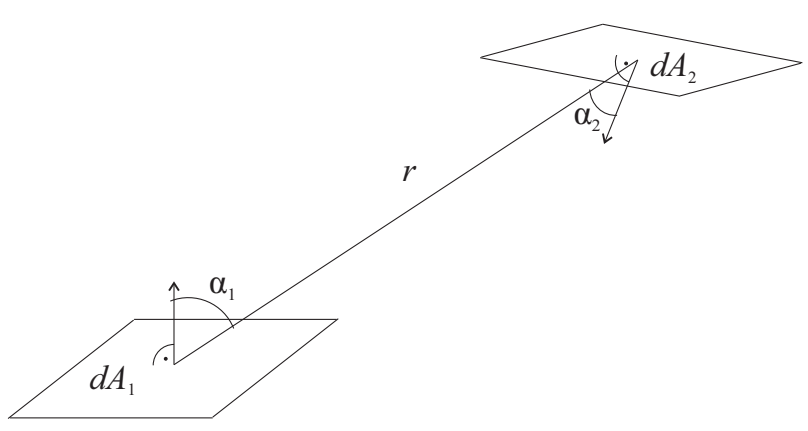

Figure 2: Infinitesimal areas and angles for the calculation of view factors if no obstruction is present.

finitesimal area element $i$ and patch $k$ can be associated with every patch as

$$
\begin{array}{r}
\Delta F_{k}=\int_{A_{j}=\text { patch } k} \frac{\cos \alpha_{i} \cos \alpha_{j}}{\pi} \mathrm{d} A_{j} \\
=\frac{\Delta \varphi}{2 \pi}\left(\cos ^{2}\left(\theta_{k}-\Delta \theta / 2\right)\right. \\
\left.\quad-\cos ^{2}\left(\theta_{k}+\Delta \theta / 2\right)\right),
\end{array}
$$

where $\Delta \theta$ and $\Delta \varphi$ are the extent in zenith and azimuth direction of the patch and it has been assumed that the hemisphere has the radius $r=1$. Following Cohen and Greenberg (1985) the view factor between an infinitesimal area $j$ and any polygon $i$ can be obtained by constructing a hemisphere with unit radius around the location of area $j$. Subsequently, polygon $i$ is projected onto this hemisphere. The view factor is then approximated by the sum of the infinitesimal view factors over those patches which are covered by the projection. With this Eq. (8) can be simplified as follows.

$$
\begin{aligned}
F_{i, j} & \approx \frac{1}{A_{i}} \sum_{k} \Delta F_{k} \int_{A_{i}} \nu_{i, j}^{k} \mathrm{~d} A_{i} \\
& =\sum_{k} \Delta F_{k} R_{i, j}^{k},
\end{aligned}
$$

where $\nu_{i, j}^{k}$ is one if the differential area on polygon $i$ "sees" polygon $j$ under the angle $\left(\theta_{k}, \varphi_{k}\right)$ and zero if it does not. $R_{i, j}^{k}$ is the ratio between the area of the region of polygon $i$ which can "see" polygon $j$ and the total area of $i$. The only approximation here is the subdivision of the hemisphere into a finite number of patches.

The implementation of this approach is straightforward. The view factors $\Delta F_{k}$ are calculated for every patch of the hemisphere. Another round of P\&C operations gives the $R_{i, j}^{k}$ and it can be used that

$$
R_{j i}^{k}=\frac{A_{i}}{A_{j}} R_{i, j}^{k^{\prime}}
$$

if $\left(\theta_{k}, \varphi_{k}\right)=-\left(\theta_{k^{\prime}}, \varphi_{k^{\prime}}\right)$. Another exclusion rule further reduces the number of necessary $\mathrm{P} \& \mathrm{C}$ operations: The condition in Eq. (5) has been used to 
identify shading surfaces, so that these are also the only surfaces that may reflect radiation onto a receiving surface. Therefore, the clipping operations to determine $R_{j i}^{k}$ have to be performed only between a receiving polygon and polygons of those surfaces that have been identified as shading surfaces for the respective polygon.

Like the irradiation matrix the view factors $F_{i, j}$ are pre-calculated, so that the large number of required clipping operations does not restrict the time resolution.

\section{Comparison to EnergyPlus calculation}

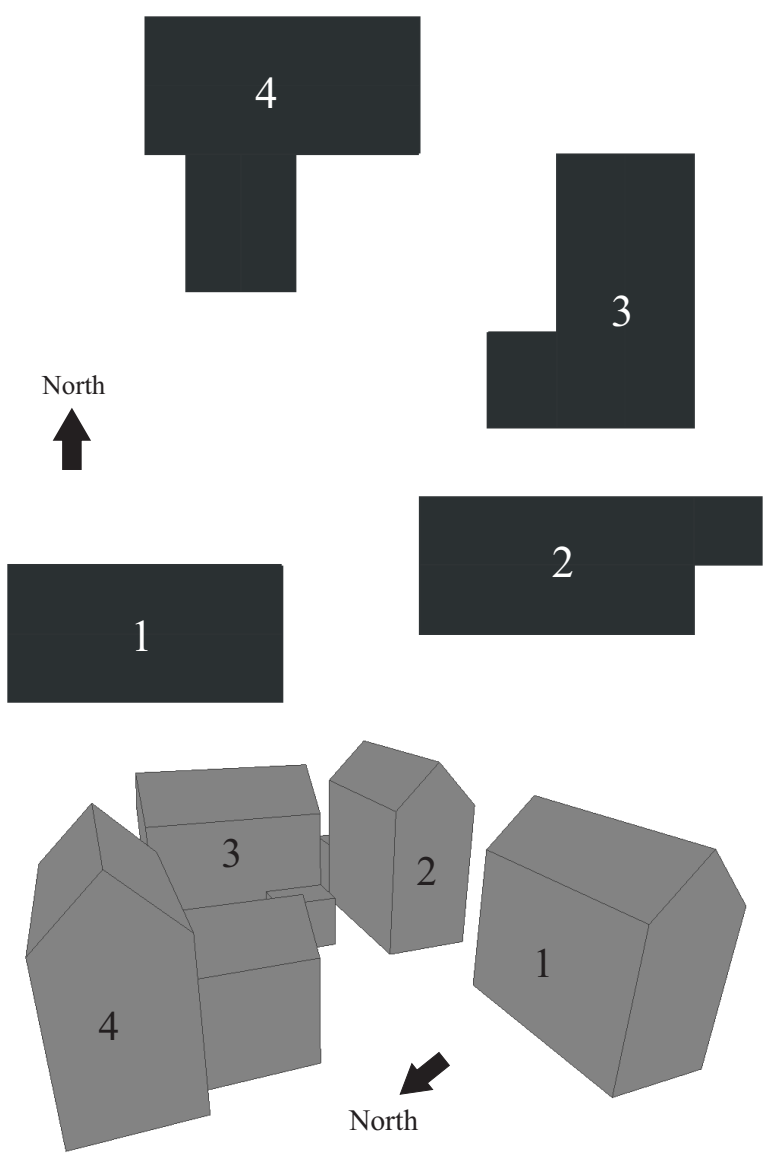

Figure 3: The reference scenario for the test of the refined PEC approach. In the top the ground surfaces of the buildings are shown, in the bottom a $3 d$ representation of the same ensemble is shown.

To test the presented approach a reference scenario is defined. It consists of four buildings which form an ensemble and stand close enough to each other to give significant shading (see Fig. 3). A ground surface which reflects sunlight can be added but is neglected in this case to reduce the complexity of the analysis. The location of the reference ensemble is chosen to be $52.47^{\circ}$ north and $13.4^{\circ}$ east, i.e. it lies in Berlin, Germany. The building ensemble is also constructed using the OpenStudio plugin of SketchUp. Building 2 is modelled as a thermal zone while the other build- ings only consist of shading surfaces. EnergyPlus is used to calculate reference values for the irradiances on the envelop surfaces of building 2. Hourly values are calculated and compared using the refined $\mathrm{P} \& \mathrm{C}$ approach and EnergyPlus.

\section{Results and discussion}

In this section the results of shading and reflection calculations using the refined $\mathrm{P} \& \mathrm{C}$ approach are presented. In a first step the reflections are turned off. In this way deviations between the refined $\mathrm{P} \& \mathrm{C}$ approach and EnergyPlus that are due to differences in the sky model can be discussed. In a second step the reflections are turned on. As weather data an IWEC file in the EnergyPlus format for Berlin, Germany, is used (ASHRAE (2001)) which contains typical weather data for one year and is derived from various years between 1982 and 1999. The results are shown for January 1st and 2nd of the resulting typical year. January 1st is a clouded day with no direct radiation while January 2nd has several hours of finite direct radiation.

To calculate the reflected contribution to the irradiance EnergyPlus uses a backward ray tracing approach with $N$ receiving points for an $N$-sided surface. From each receiving point 90 rays are sent out which may hit obstructions or the sky US DOE (2007). In particular, the limited number of receiving points on potentially large surfaces can cause errors which are expected not to be present in the refined P\&C approach.

\section{Without reflections}

Figure 4 shows the irradiance obtained by the refined $\mathrm{P} \& \mathrm{C}$ calculations and by EnergyPlus for the main north, east, south, and west facade of building 2 . In all cases the qualitative behavior of the irradiances agrees very well. The highest deviation on the first day is found on the east wall around noon with approximately $9 \%$. On the second day absolute and relative deviations increase. Especially in the morning hours the deviations go up to $50 \%$. At noon the highest relative deviation is found to be around $10 \%$ on the south wall.

The weather file which is used by both programs contains hourly values for direct, diffuse, etc. radiation. These have been calculated as averages over measurements of the previous respective hour. While the refined P\&C calculations use these values directly, EnergyPlus performs an interpolation to approximate the correct values at the full hour (US DOE (2007)). This most likely explains the strong deviations in the morning hours of the second day because here the direct radiation changes rapidly.

Another source of deviations are the different sky models that the two approaches use. For the refined $\mathrm{P} \& \mathrm{C}$ approach the model for the angular distribution of luminance by Perez et al. (1993) is used with 

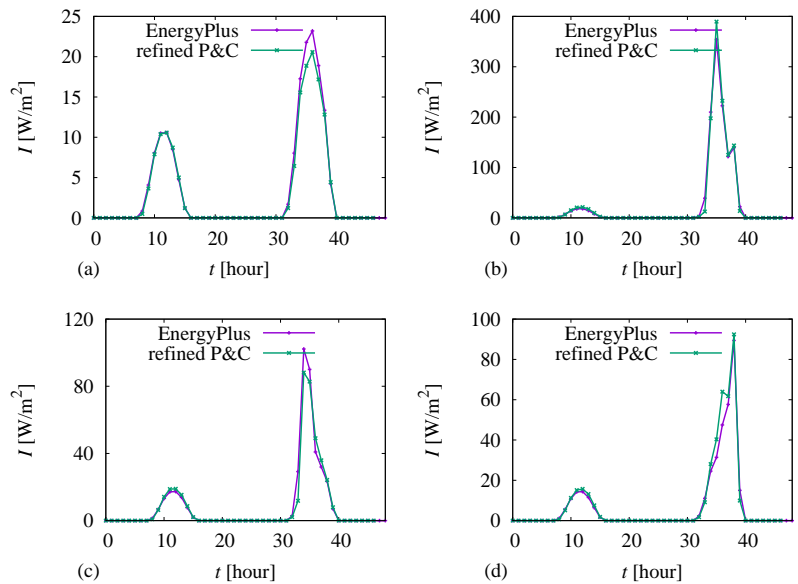

Figure 4: Irradiances for January 1st and 2nd on (a) the north surface, (b) the south surface, (c) the east surface, and (d) the west surface of the main part of building 2. The irradiances are calculated without taking reflections into account.

a normalization factor to calculate an angular distribution of radiances. EnergyPlus relies on the model for diffuse irradiance on tilted surfaces by Perez et al. (1990) with more precise model coefficients from 1999 (US DOE (2007)).

\section{With reflections}

When the refined $\mathrm{P} \& \mathrm{C}$ approach is applied to the reference scenario the sum in Equation 7 runs over the original envelop surfaces. This choice implies an additional approximation. It corresponds to the assumption of a uniform sky which EnergyPlus applies in the ray tracing approach to determine the reflected sky diffuse radiation. Figure 5 shows the difference in irradiance between calculations with and without reflections $\Delta I$. Again, the qualitative behavior agrees very well. E.g., the change of the maximum of irradiance on the second day from $12 \mathrm{pm}$ to 11 am on the north wall is present in both outcomes. There appears to be a systematic tendency of the refined $\mathrm{P} \& \mathrm{C}$ approach to give a higher reflected contribution to the irradiance than EnergyPlus - especially when there is significant direct solar radiation. This leads to a partial compensation of the deviation in the total irradiance between the two approaches on the north and east surface but increases the deviation on the west surface. The relative deviations in the reflected contribution to the irradiance tend to be higher than for the irradiance without reflections. Whether this effect is due to the limited number of receiving points in the ray tracing which is performed by EnergyPlus is hard to determine in the given scenario.

A possible additional source for deviations in the reflected part is the fact that EnergyPlus treats the direct and sky diffuse solar radiation separately while the refined $\mathrm{P} \& \mathrm{C}$ approach does not distinguish between them. The separate treatment of the direct so-
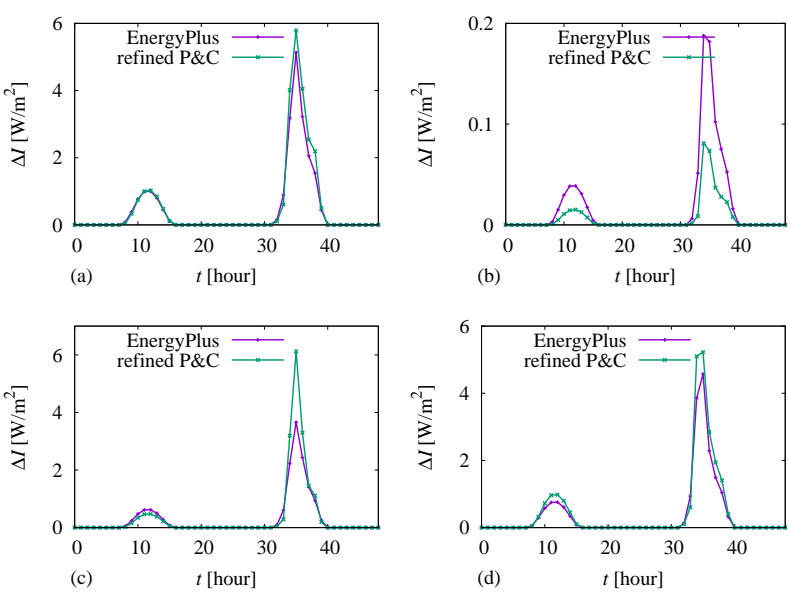

Figure 5: Irradiance $\Delta I$ reflected onto (a) the north surface, (b) the south surface, (c) the east surface, and (d) the west surface of the main part of building 2 for January 1st and 2 nd.

lar radiation allows EnergyPlus to consider obstructions between the sun position and a reflecting point that is hit by a ray coming from a receiving point. The approximation to let the sum in Equation 7 only run over the original building surfaces effectively means that all the radiation that reaches a surface is uniformly distributed over this surface and then reflected by it. This can lead to an under- or overestimation of reflected irradiance. To verify whether this really explains the higher reflected irradiance values in the refined $\mathrm{P} \& \mathrm{C}$ approach further investigations are required.

A thorough optimization of the code has not been performed, yet. However, for the short period of 48 hours the part of the program that has to be executed only once clearly gives the dominant contribution to the computation time. The repeated hourly calculations add up to $\sim 10^{-2} \mathrm{~s}$ and are, therefore, of the same order of magnitude as EnergyPlus.

\section{Conclusions}

In this paper a refined $\mathrm{P} \& \mathrm{C}$ approach to calculate the solar irradiance on building surfaces is presented. It takes the direct, sky diffuse, and reflected radiation into account. It extends known concepts of solar shading calculation and the calculation of reflections. It has been applied to a reference scenario of a small ensemble of buildings and its results have been compared to the outcome of a simulation in EnergyPlus. Calculations with and without reflections give good qualitative agreement between the results of the refined P\&C approach and EnergyPlus. The quantitative deviations in the peak radiance hours around noon are of the order of $10 \%$ without reflections. The source of these deviations can most likely be found in the different models for sky diffuse radiation.

The reflected contribution to the irradiance also 
shows a good qualitative agreement but higher quantitative deviations. The lack of a measured true irradiance value does not allow for a clear decision which result is closer to reality. However, in the refined $\mathrm{P} \& \mathrm{C}$ approach an additional approximation was applied to reduce the size of the view factor matrix. This makes it plausible that the reflected contribution to the irradiance is overestimated in some cases.

Since the irradiation matrix and the view factor matrix are calculated only once for every time series the refined $\mathrm{P} \& \mathrm{C}$ approach shows an acceptable performance and does not restrict time resolution because the irradiation matrix and the view factors can be pre-calculated. An additional speed-up is expected with a further optimization of the code.

To summarize: The refined $\mathrm{P} \& \mathrm{C}$ approach gives good qualitative and in some cases even quantitative agreement with the widely used and tested building energy simulation software EnergyPlus. The advantages of such a $\mathrm{P} \& \mathrm{C}$ approach are that fewer approximations have to be made than in ray tracing and pixel counting methods. It has been shown that $\mathrm{P} \& \mathrm{C}$ approaches, nevertheless, can give acceptable performance and that there is still room for a reduction of computation times. However, more work is necessary to fully understand the strengths and weaknesses of the presented approach. To further examine the deviations between the reflected contribution in the refined P\&C approach and EnergyPlus the effect of the approximation that the irradiance is evenly distribute over a building surface before reflections are calculated has to be studied in more detail. It may also be benefitial to introduce a separation of direct and sky diffuse radiation in the calculation of reflections. An experimentally determined "ground truth" of irradiance values is desirable to determine the accuracy of the refined P\&C approach.

\section{Acknowledgment}

We acknowledge funding by the German Federal Ministry for Economic Affairs and Energy for this work.

\section{Nomenclature}

$A_{i} \quad$ area of polygon $i\left(\mathrm{~m}^{2}\right)$

$F_{i, j} \quad$ view factor from polygon $i$ to polygon $j$

$\Delta F_{k} \quad$ infenitesimal view factor associated with a patch of the hemisphere $k$

$I_{\mathrm{dh}} \quad$ diffuse horizontal radiation $\left(\mathrm{W} \mathrm{m}^{-2}\right)$

$I^{n} \quad$ normal irradiance $\left(\mathrm{W} \mathrm{m}^{-2}\right)$

$I_{n, k}^{0} \quad$ irradiance on surface $n$ from sky patch $k$ $\left(\mathrm{W} \mathrm{m}^{-2}\right)$

$\Delta I \quad$ difference in irradiance $\left(\mathrm{W} \mathrm{m}^{-2}\right)$

$i, j \quad$ indices for polygons on buidling surfaces that arise from $\mathrm{P} \& \mathrm{C}$ operations

$k \quad$ index for sky patches

$l \quad$ luminance distribution $\left(\mathrm{cd} \mathrm{m}^{-2} \mathrm{sr}^{-1}\right)$

$M \quad$ irradiation matrix

$m_{i, k} \quad$ irradiation factor

$n \quad$ index for building surfaces

$\mathbf{n}_{n} \quad$ norm vector on surface $n$

$\mathbf{n}_{\text {sky }}$ norm vector pointing into the direction of incoming radiation from the sky dome

$R_{\mathrm{d}}(\theta, \gamma)$ radiance distribution $\left(\mathrm{W} \mathrm{m}^{-2} \mathrm{sr}^{-1}\right)$

$R_{i, j}^{k} \quad$ ratio between the are of the region of polygon $i$ that can see polygon $j$ and the total area of polygon $i$

$r \quad$ distance between two infinitesimal area elements

\section{Greek symbols}

$\alpha_{i} \quad$ angle between normal vector of an infinitesimal area element and the vector that connects it to another infinitesimal area element

$\gamma \quad$ angle between the center of a sky patch and the sun

$\theta \quad$ zenith angle in spherical coordinates

$\Delta \theta \quad$ extent of a hemisphere patch in zenith direction

$\varphi \quad$ azimuth angle in spherical coordinates

$\Delta \varphi \quad$ extent of a hemisphere patch in azimuth direction

$\chi \quad$ irradiance normalization factor

$\nu_{i, j} \quad$ takes the value 1 if a differential area on polygon $i$ can see polygon $j$ under a given angle and the value 0 if not

$\nu_{i, j}^{k} \quad$ takes the value 1 if a differential area on polygon $i$ can see polygon $j$ under $\left(\theta_{k}, \varphi_{k}\right)$ and the value 0 if not

$\Delta \Omega_{k} \quad$ solid angle covered by sky patch $k(\mathrm{sr})$ $\rho_{i} \quad$ reflectance

\section{References}

American Society of Heating, Refrigerating and Air-Conditioning Engineers (2001). International Weather for Energy Calculations (IWEC Weather Files) Users Manual and CD-ROM.

Atherton, P., K. Weiler, and D. Greenberg (1978). Polygon shadow generation. ACM SIGGRAPH Computer Graphics 12(3), 275-281.

Bretagnon, P. and G. Francou (1988). Planetary theories in rectangular and spherical variables - vsop 
87 solutions. Astronomy and Astrophysics 202(12), 309-315.

Catita, C., P. Redweik, J. Pereira, and M. Brito (2014). Extending solar potential analysis in buildings to vertical facades. Computers \& Geosciences $66,1-12$.

Cohen, M. F. and D. P. Greenberg (1985). The hemicube: A radiosity solution for complex environments. SIGGRAPH Comput. Graph. 19(3), 31-40.

Crawley, D. B., J. W. Hand, M. Kummert, and B. T. Griffith (2008). Contrasting the capabilities of building energy performance simulation programs. Building and Environment 43(4), 661 - 673. Part Special: Building Performance Simulation.

de Almeida Rocha, A. P., R. C. Oliveira, and N. Mendes (2017). Experimental validation and comparison of direct solar shading calculations within building energy simulation tools: Polygon clipping and pixel counting techniques. Solar Energy 158, 462-473.

Estevam Schmiedt, J., D. Cerra, D. Dahlke, S. Dill, N. Ge, J. Göttsche, A. Haas, U. Heiden, M. Israel, F. Kurz, et al. (2017). Remote sensing techniques for building models and energy performance studies of buildings. Volume 71.

Freitas, S., C. Catita, P. Redweik, and M. Brito (2015). Modelling solar potential in the urban environment: State-of-the-art review. Renewable and Sustainable Energy Reviews 41, 915-931.

Frommholz, D., M. Linkiewicz, H. Meissner, D. Dahlke, and A. Poznanska (2015). Extracting semantically annotated $3 \mathrm{~d}$ building models with textures from oblique aerial imagery. The International Archives of Photogrammetry, Remote Sensing and Spatial Information Sciences 40(3), 53.

Han, Y., J. E. Taylor, and A. L. Pisello (2017). Exploring mutual shading and mutual reflection interbuilding effects on building energy performance. Applied Energy 185, 1556 - 1564. Clean, Efficient and Affordable Energy for a Sustainable Future.

Igawa, N., Y. Koga, T. Matsuzawa, and H. Nakamura (2004). Models of sky radiance distribution and sky luminance distribution. Solar Energy $77(2), 137-$ 157.

Kim, C.-H. and K.-S. Kim (2015). Evaluation of thermal and visual environment for the glazing and shading device in an office building with installed of venetian blind. KIEAE Journal 15(6), 101-109.

Liao, W. and Y. Heo (2017). A simplified vectorbased method for irradiance prediction at urban scale. In Proceedings of the 15th IBPSA Conference, pp. 2388-2397.
Maestre, I., L. Pérez-Lombard, J. Foncubierta, and P. Cubillas (2013). Improving direct solar shading calculations within building energy simulation tools. Journal of Building Performance Simulation 6(6), 437-448.

Melo, E. G., M. P. Almeida, R. Zilles, and J. A. Grimoni (2013). Using a shading matrix to estimate the shading factor and the irradiation in a threedimensional model of a receiving surface in an urban environment. Solar Energy 92, 15-25.

Niewienda, A. and F. Heidt (1996). SOMBRERO: A PC-tool to calculate shadows on arbitrarily oriented surfaces. Solar Energy 58(4), 253 - 263. Selected Proceedings of the ISES 1995: Solar World Congress. Part II.

Open Geospatial Consortium (2012). OGC City Geography Markup Language (CityGML) Encoding Standard (OGC 12-019).

Perez, R., P. Ineichen, R. Seals, J. Michalsky, and R. Stewart (1990). Modeling daylight availability and irradiance components from direct and global irradiance. Solar Energy 44(5), 271-289.

Perez, R., R. Seals, and J. Michalsky (1993). All-weather model for sky luminance distribution-preliminary configuration and validation. Solar energy 50(3), 235-245.

Redweik, P., C. Catita, and M. Brito (2013). Solar energy potential on roofs and facades in an urban landscape. Solar Energy 97, 332-341.

Robinson, D. and A. Stone (2004a). Irradiation modelling made simple: the cumulative sky approach and its applications. In Plea2004 - The 21st Conference on Passive and Low Energy Architecture. Eindhoven (Netherlands), 19 - 22 September 2004.

Robinson, D. and A. Stone (2004b). Solar radiation modelling in the urban context. Solar Energy $77(3)$, 295-309.

Tregenza, P. R. (1987). Subdivision of the sky hemisphere for luminance measurements. Lighting Research \& Technology 19(1), 13-14.

US Department of Energy (2007). EnergyPlus Documentation, Engineering Reference.

Verma, V., R. Kumar, and S. Hsu (2006, June). 3d building detection and modeling from aerial lidar data. In 2006 IEEE Computer Society Conference on Computer Vision and Pattern Recognition (CVPR'06), Volume 2, pp. 2213-2220. New York (USA), 17-22 June 2006. 